\title{
Algebraically grid-like graphs have large tree-width
}

\author{
Daniel Weißauer \\ Department of Mathematics \\ University of Hamburg \\ Hamburg, Germany \\ daniel.weissauer@gmail.com
}

Submitted: Feb 27, 2018; Accepted: Oct 15, 2018; Published: Jan 25, 2019

(C) The author. Released under the CC BY license (International 4.0).

\begin{abstract}
By the Grid Minor Theorem of Robertson and Seymour, every graph of sufficiently large tree-width contains a large grid as a minor. Tree-width may therefore be regarded as a measure of 'grid-likeness' of a graph.

The grid contains a long cycle on the perimeter, which is the $\mathbb{F}_{2}$-sum of the rectangles inside. Moreover, the grid distorts the metric of the cycle only by a factor of two. We prove that every graph that resembles the grid in this algebraic sense has large tree-width:

Let $k, p$ be integers, $\gamma$ a real number and $G$ a graph. Suppose that $G$ contains a cycle of length at least $2 \gamma p k$ which is the $\mathbb{F}_{2}$-sum of cycles of length at most $p$ and whose metric is distorted by a factor of at most $\gamma$. Then $G$ has tree-width at least $k$.
\end{abstract}

Mathematics Subject Classifications: 05C83, 05C12, 05C38

\section{Introduction}

The edge space of a graph $G=(V, E)$ is the vector space over the 2-element field $\mathbb{F}_{2}$ consisting of all maps $E \rightarrow \mathbb{F}_{2}$, with addition defined pointwise as $(f \oplus g)(e)=f(e)+g(e)$. Each such map $f: E \rightarrow \mathbb{F}_{2}$ may be identified with the set of all $e \in E$ for which $f(e)=1$. Addition of maps then corresponds to symmetric difference of sets of edges, and we call $F_{1} \oplus F_{2}:=F_{1} \Delta F_{2}$ the $\mathbb{F}_{2}$-sum of $F_{1}, F_{2} \subseteq E$.

The cycle space of $G$ is the subspace of the edge space which is generated by the (edge sets of) cycles of $G$. In this context, we do not formally distinguish between a cycle $C \subseteq G$ and its set of edges $E(C) \subseteq E$ and simply speak of the $\mathbb{F}_{2}$-sum of cycles. See the book by Diestel [5] for more background on the cycle space.

For a positive integer $n$, the $(n \times n)$-grid is the graph $G_{n}$ whose vertices are all pairs $(i, j)$ with $1 \leqslant i, j \leqslant n$, where two points are adjacent when they are at Euclidean 
distance 1 . The cycle $C_{n}$, which bounds the outer face in the natural drawing of $G_{n}$ in the plane, has length $4(n-1)$ and is the $\mathbb{F}_{2}$-sum of the rectangles bounding the inner faces. This is by itself not a distinctive feature of graphs with large tree-width: The situation is similar for the $n$-wheel $W_{n}$, the graph consisting of a cycle $D_{n}$ of length $n$ and a vertex $x \notin D_{n}$ which is adjacent to every vertex of $D_{n}$. There, $D_{n}$ is the $\mathbb{F}_{2}$-sum of all triangles $x y z$ for $y z \in E\left(D_{n}\right)$. Still, $W_{n}$ only has tree-width 3 .

The key difference is the fact that in the wheel, the metric of the cycle is heavily distorted: any two vertices of $D_{n}$ are at distance at most two within $W_{n}$, even if they are far apart within $D_{n}$. In the grid, however, the distance between two vertices of $C_{n}$ within $G_{n}$ is at least half of their distance within $C_{n}$.

In order to incorporate this factor of two and to allow for more flexibility, we equip the edges of our graphs with lengths. For a graph $G$, a length-function on $G$ is simply a map $\ell: E(G) \rightarrow \mathbb{R}_{>0}$. We then define the $\ell$-length $\ell(H)$ of a subgraph $H \subseteq G$ as the sum of the lengths of all edges of $H$. This naturally induces a notion of distance between two vertices of $G$, where we define $d_{G}^{\ell}$ as the minimum $\ell$-length of a path containing both. A subgraph $H \subseteq G$ is $\ell$-geodesic if it contains a path of length $d_{G}^{\ell}(a, b)$ between any two vertices $a, b \in V(H)$.

When no length-function is specified, the notions of length, distance and geodecity are to be read with respect to $\ell \equiv 1$ constant.

On the grid-graph $G_{n}$, consider the length-function $\ell$ which is equal to 1 on $E\left(C_{n}\right)$ and assumes the value 2 elsewhere. Then $C_{n}$ is $\ell$-geodesic of length $\ell\left(C_{n}\right)=4(n-1)$ and the sum of cycles of $\ell$-length at most 8 . We show that any graph which shares this algebraic feature has large tree-width.

Theorem 1. Let $k$ be a positive integer and $r>0$. Let $G$ be a graph with rational-valued length-function $\ell$. Suppose $G$ contains an $\ell$-geodesic cycle $C$ with $\ell(C) \geqslant 2$ rk, which is the $\mathbb{F}_{2}$-sum of cycles of $\ell$-length at most $r$. Then the tree-width of $G$ is at least $k$.

In Section 3, we prove a qualitative converse to Theorem 1, showing that graphs of large tree-width are 'algebraically grid-like'.

The starting point of Theorem 1 was a similar result of Matthias Hamann and the author [2]. There, it is assumed that not only the fixed cycle $C$, but the whole cycle space of $G$ is generated by short cycles.

Theorem 2 ([2, Corollary 3]). Let $k, p$ be positive integers. Let $G$ be a graph whose cycle space is generated by cycles of length at most $p$. If $G$ contains a geodesic cycle of length at least $k p$, then the tree-width of $G$ is at least $k$.

It should be noted that Theorem 2 is not implied by Theorem 1 , as the constant factors are different. In fact, the proofs are also quite different, although Lemma 5 below was inspired by a similar parity-argument in [2].

It is tempting to think that, conversely, Theorem 1 could be deduced from Theorem 2 by adequate manipulation of the graph $G$, but we have not been successful with such attempts. 


\section{Proof of Theorem 1}

The relation to tree-width is established via a well-known separation property of graphs of bounded tree-width, due to Robertson and Seymour [3].

Lemma 3 ([3]). Let $k$ be a positive integer, $G$ a graph and $A \subseteq V(G)$. If the tree-width of $G$ is less than $k$, then there exists $X \subseteq V(G)$ with $|X| \leqslant k$ such that every component of $G-X$ contains at most $\frac{1}{2}|A \backslash X|$ vertices of $A$.

It is not hard to see that Theorem 1 can be reduced to the case where $\ell \equiv 1$. This case is treated in the next theorem.

Theorem 4. Let $k, p$ be positive integers. Let $G$ be a graph containing a geodesic cycle $C$ of length at least $4\lfloor p / 2\rfloor k$, which is the $\mathbb{F}_{2}$-sum of cycles of length at most $p$. Then for every $X \subseteq V(G)$ of order at most $k$, some component of $G-X$ contains at least half the vertices of $C$.

Proof of Theorem 1, assuming Theorem 4. Let $\mathcal{D}$ be a set of cycles of $\ell$-length at most $r$ with $C=\bigoplus \mathcal{D}$. Since $\ell$ is rational-valued and the premise also holds for $r^{\prime}$ the maximum $\ell$-length of a cycle in $\mathcal{D}$, we may assume that $r \in \mathbb{Q}$. Take an integer $M$ so that $r M$ and $\ell^{\prime}(e):=M \ell(e)$ are natural numbers for every $e \in E(G)$.

Obtain the subdivision $G^{\prime}$ of $G$ by replacing every $e \in E(G)$ by a path of length $\ell^{\prime}(e)$. Denote by $C^{\prime}, D^{\prime}$ the subdivisions of $C$ and $D$ for every $D \in \mathcal{D}$, respectively. Then $C^{\prime}=\bigoplus_{D \in \mathcal{D}} D^{\prime}$ and $\left|C^{\prime}\right|=M \ell(C) \geqslant 2(M r) k$, while $\left|D^{\prime}\right|=M \ell(D) \leqslant M r$ for every $D \in \mathcal{D}$.

Assume for a contradiction that the tree-width of $G$ was less than $k$. Since tree-width is invariant under subdivision, the tree-width of $G^{\prime}$ is also less than $k$. By Lemma 3, there exists a set $X \subseteq V\left(G^{\prime}\right)$ with $|X| \leqslant k$ such that every component of $G^{\prime}-X$ contains at most $\frac{1}{2}\left|V\left(C^{\prime}\right) \backslash X\right|$ vertices of $V\left(C^{\prime}\right)$. Since $C^{\prime}$ is a connected subgraph of $G^{\prime}, X$ must contain at least one vertex of $C^{\prime}$. By Theorem 4 , there is a component $K$ of $G^{\prime}-X$ that contains at least half the vertices of $C^{\prime}$. But then

$$
\frac{1}{2}\left|C^{\prime}\right| \leqslant\left|K \cap V\left(C^{\prime}\right)\right| \leqslant \frac{1}{2}\left|V\left(C^{\prime}\right) \backslash X\right|<\frac{1}{2}\left|C^{\prime}\right|,
$$

which is a contradiction.

Our goal is now to prove Theorem 4. The proof consists of two separate lemmas. The first lemma involves separators and $\mathbb{F}_{2}$-sums of cycles.

Lemma 5. Let $G$ be a graph, $C \subseteq G$ a cycle and $\mathcal{D}$ a set of cycles in $G$ such that $C=\bigoplus \mathcal{D}$. Let $\mathcal{R}$ be a set of disjoint vertex-sets of $G$ such that for every $R \in \mathcal{R}, R \cap V(C)$ is either empty or induces a connected subgraph of $C$. Then either some $D \in \mathcal{D}$ meets two distinct $R, R^{\prime} \in \mathcal{R}$ or there is a component $Q$ of $G-\bigcup \mathcal{R}$ with $V(C) \subseteq V(Q) \cup \bigcup \mathcal{R}$.

Proof. Suppose that no $D \in \mathcal{D}$ meets two distinct $R, R^{\prime} \in \mathcal{R}$. Then $C$ has no edges between the sets in $\mathcal{R}$, since any such edge would have to lie in at least one $D \in \mathcal{D}$. Let $Y:=\bigcup \mathcal{R}$ and let $\mathcal{Q}$ be the set of components of $G-Y$. 
We claim that for all $Q \in \mathcal{Q}, R \in \mathcal{R}$ and $D \in \mathcal{D}$, the number of edges of $D$ between $Q$ and $R$ is even. This is trivial if $D$ has no edges between $Q$ and $R$. Otherwise, $D$ meets $R$ and thus cannot meet $Y \backslash R$. Therefore, all edges of $D$ between $Q$ and $V(G) \backslash Q$ must join $Q$ to $R$. As $D$ is a cycle, it has an even number of edges between $Q$ and $V(G) \backslash Q$ and thus between $Q$ and $R$. This finishes the proof of the claim.

Now, as $C=\bigoplus \mathcal{D}$, we find that that for all $Q \in \mathcal{Q}, R \in \mathcal{R}$, the number of edges of $C$ between $Q$ and $R$ is

$$
e_{C}(Q, R) \equiv \sum_{D \in \mathcal{D}} e_{D}(Q, R) \equiv 0 \quad \bmod 2
$$

For every $R \in \mathcal{R}$ which intersects $C$, there are precisely two edges of $C$ between $R$ and $V(C) \backslash R$, because $R \cap C$ is connected. As mentioned above, $C$ contains no edges between $R$ and $Y \backslash R$, so both edges join $R$ to $V(G) \backslash Y$. But $C$ has an even number of edges between $R$ and each component of $V(G) \backslash Y$, so it follows that both edges join $R$ to the same $Q \in \mathcal{Q}$.

Since every component of $C-(C \cap Y)$ is contained in a component of $G-Y$, it follows that there is a $Q \in \mathcal{Q}$ containing all vertices of $C$ not contained in $Y$.

To deduce Theorem 4, we want to apply Lemma 5 to a suitable family $\mathcal{R}$ with $\bigcup \mathcal{R} \supseteq X$ to deduce that some component of $G-X$ contains many vertices of $C$. Here, $\mathcal{D}$ consists of cycles of length at most $\ell$, so if the sets in $\mathcal{R}$ are at pairwise distance $>\lfloor\ell / 2\rfloor$, then no $D \in \mathcal{D}$ can pass through two of them. The next lemma ensures that we can find such a family $\mathcal{R}$ with a bound on $|\bigcup \mathcal{R}|$, when the cycle $C$ is geodesic.

Lemma 6. Let d be a positive integer, $G$ a graph, $X \subseteq V(G)$ and $C \subseteq G$ a geodesic cycle. Then there exists a family $\mathcal{R}$ of disjoint sets of vertices of $G$ with $X \subseteq \cup \mathcal{R} \subseteq X \cup V(C)$ and $|V(C) \cap \bigcup \mathcal{R}| \leqslant 2 d|X|$ such that for each $R \in \mathcal{R}$, the set $V(C) \cap R$ induces a (possibly empty) connected subgraph of $C$ and the distance between any two sets in $\mathcal{R}$ is greater than $d$.

Proof. Let $Y \subseteq V(G)$ and $y \in Y$. For $j \geqslant 0$, let $B_{Y}^{j}(y)$ be the set of all $z \in Y$ at distance at most $j d$ from $y$. Since $\left|B_{Y}^{0}(y)\right|=1$, there is a maximum number $j$ for which $\left|B_{Y}^{j}(y)\right| \geqslant 1+j$, and we call this $j=j_{Y}(y)$ the range of $y$ in $Y$. Observe that every $z \in Y \backslash B_{Y}^{j_{Y}(y)}$ has distance greater than $\left(j_{Y}(y)+1\right) d$ from $y$.

Starting with $X_{1}:=X$, repeat the following procedure for $k \geqslant 1$. If $X_{k}$ does not meet $C$, terminate the process. Otherwise, pick an $x_{k} \in X_{k} \cap V(C)$ of maximum range in $X_{k}$. Let $j_{k}:=j_{X_{k}}\left(x_{k}\right)$ and $B_{k}:=B_{X_{k}}^{j_{k}}\left(x_{k}\right)$. Define $X_{k+1}:=X_{k} \backslash B_{k}$ and repeat.

Since the size of $X_{k}$ decreases in each step, there is a smallest integer $m$ for which $X_{m+1} \cap V(C)$ is empty, at which point the process terminates. By construction, the distance between $B_{k}$ and $X_{k+1}$ is greater than $d$ for each $k \leqslant m$. For each $k$ satisfying $1 \leqslant k \leqslant m$, there are two edge-disjoint paths $P_{k}^{1}, P_{k}^{2} \subseteq C$, starting at $x_{k}$ and each of length at most $j_{k} d$, so that $B_{k} \cap V(C) \subseteq S_{k}:=P_{k}^{1} \cup P_{k}^{2}$, because $C$ is geodesic. Choose these paths minimal, so that the endvertices of $S_{k}$ lie in $B_{k}$. Note that every vertex of $S_{k}$ has distance at most $j_{k} d$ from $x_{k}$. Therefore, the distance between $R_{k}:=B_{k} \cup S_{k}$ and $X_{k+1}$ is greater than $d$. 
We claim that the distance between $R_{k}$ and $R_{k^{\prime}}$ is greater than $d$ for any $k<k^{\prime}$. Since $B_{k^{\prime}} \subseteq X_{k+1}$, it is clear that every vertex of $B_{k^{\prime}}$ has distance greater than $d$ from $R_{k}$. Take a vertex $q \in S_{k^{\prime}} \backslash B_{k^{\prime}}$ and assume for a contradiction that its distance to $R_{k}$ was at most $d$. Then the distance between $x_{k}$ and $q$ is at most $\left(j_{k}+1\right) d$. Let $a, b \in B_{k^{\prime}}$ be the endvertices of $S_{k^{\prime}}$. If $x_{k} \notin S_{k^{\prime}}$, then one of $a$ and $b$ lies on the shortest path from $x_{k}$ to $q$ within $C$. Since $C$ is geodesic, that endvertex then has distance at most $\left(j_{k}+1\right) d$ from $x_{k}$. But then, since $j_{k}$ is the range of $x_{k}$ in $X_{k}$, that vertex would already lie in $B_{k}$, a contradiction. Suppose now that $x_{k} \in S_{k^{\prime}}$. Then $x_{k}$ lies on one of $P_{k^{\prime}}^{1}$ or $P_{k^{\prime}}^{2}$, so the distance between $x_{k}$ and $x_{k^{\prime}}$ is at most $j_{k^{\prime}} d$. Since $x_{k^{\prime}} \in X_{k} \cap V(C)$, it follows from our choice of $x_{k}$ that

$$
j_{k}=j_{X_{k}}\left(x_{k}\right) \geqslant j_{X_{k}}\left(x_{k^{\prime}}\right) \geqslant j_{X_{k^{\prime}}}\left(x_{k^{\prime}}\right)=j_{k^{\prime}}
$$

where the second inequality follows from the fact that $X_{k^{\prime}} \subseteq X_{k}$ and $j_{Y}(y) \geqslant j_{Y^{\prime}}(y)$ whenever $Y \supseteq Y^{\prime}$. But then $x_{k^{\prime}} \in B_{k}$, a contradiction. This finishes the proof of the claim.

Finally, let $\mathcal{R}:=\left\{R_{k}: 1 \leqslant k \leqslant m\right\} \cup\left\{X_{m+1}\right\}$. The distance between any two sets in $\mathcal{R}$ is greater than $d$. For $k \leqslant m, R_{k} \cap V(C)=S_{k}$ is a connected subgraph of $C$, while $X_{m+1} \cap V(C)$ is empty. Moreover,

$$
\begin{aligned}
|V(C) \cap \bigcup \mathcal{R}| & =\sum_{k=1}^{m}\left|S_{k}\right| \leqslant \sum_{k=1}^{m}\left(1+2 j_{k} d\right) \leqslant \sum_{k=1}^{m}\left(1+2\left(\left|B_{k}\right|-1\right) d\right) \\
& \leqslant \sum_{k=1}^{m} 2\left|B_{k}\right| d \leqslant 2 d|X| .
\end{aligned}
$$

Proof of Theorem 4. Let $X \subseteq V(G)$ be of order at most $k$ and let $d:=\lfloor p / 2\rfloor$. By Lemma 6 , there exists a family $\mathcal{R}$ of disjoint sets of vertices of $G$ with $X \subseteq \bigcup \mathcal{R} \subseteq$ $X \cup V(C)$ and $|V(C) \cap \bigcup \mathcal{R}| \leqslant 2 d k$ so that for each $R \in \mathcal{R}$, the set $R \cap V(C)$ induces a (possibly empty) connected subgraph of $C$ and the distance between any two sets in $\mathcal{R}$ is greater than $d$.

Let $\mathcal{D}$ be a set of cycles of length at most $p$ with $C=\bigoplus \mathcal{D}$. Then no $D \in \mathcal{D}$ can meet two distinct $R, R^{\prime} \in \mathcal{R}$, since the diameter of $D$ is at most $d$. By Lemma 5 , there is a component $Q$ of $G-\bigcup \mathcal{R}$ which contains every vertex of $C \backslash \bigcup \mathcal{R}$. This component is connected in $G-X$ and therefore contained in some component $Q^{\prime}$ of $G-X$, which then satisfies

$$
\left|Q^{\prime} \cap V(C)\right| \geqslant|C|-|V(C) \cap \bigcup \mathcal{R}| \geqslant|C|-2 d k .
$$

Since $|C| \geqslant 4 d k$, the claim follows.

\section{Remarks}

We have described the content of Theorem 1 as an algebraic criterion for a graph to have large tree-width. The reader might object that the cycle $C$ being $\ell$-geodesic is a metric property and not an algebraic one. Karl Heuer has pointed out to us, however, 
that geodecity of a cycle can be expressed as an algebraic property after all. This is a consequence of a more general lemma of Gollin and Heuer [1], which allowed them to introduce a meaningful notion of geodecity for cuts.

Proposition 7 ([1]). Let $G$ be a graph with length-function $\ell$ and $C \subseteq G$ a cycle. Then $C$ is $\ell$-geodesic if and only if there do not exist cycles $D_{1}, D_{2}$ with $\ell\left(D_{1}\right), \ell\left(D_{2}\right)<\ell(C)$ such that $C=D_{1} \oplus D_{2}$.

Finally, we would like to point out that Theorem 1 does not only offer a 'one-way criterion' for large tree-width, but that it has a qualitative converse. First, we recall the Grid Minor Theorem of Robertson and Seymour [4], phrased in terms of walls. For a positive integer $t$, an elementary $t$-wall is the graph obtained from the $2 t \times t$-grid as follows. Delete all edges with endpoints $(i, j),(i, j+1)$ when $i$ and $j$ have the same parity. Delete the two resulting vertices of degree one. A $t$-wall is any subdivision of an elementary $t$-wall. Note that the $(2 t \times 2 t)$-grid has a subgraph isomorphic to a $t$-wall.

Theorem 8 (Grid Minor Theorem [4]). For every $t \in \mathbb{N}$ there exists a $k \in \mathbb{N}$ such that every graph of tree-width at least $k$ contains a $t$-wall.

Here, then, is our qualitative converse to Theorem 1, showing that the algebraic condition in the premise of Theorem 1 in fact captures tree-width.

Corollary 9. For every $L \in \mathbb{N}$ there exists a $k \in \mathbb{N}$ such that for every graph $G$ the following holds. If $G$ has tree-width at least $k$, then there exists a rational-valued lengthfunction on $G$ such that $G$ contains an $\ell$-geodesic cycle $C$ with $\ell(C) \geqslant L$ which is the $\mathbb{F}_{2}$-sum of cycles of $\ell$-length at most 1 .

Proof. Let $s:=6 L$. By the Grid Minor Theorem, there exists an integer $k$ such that every graph of tree-width at least $k$ contains an $s$-wall. Suppose $G$ is a graph of tree-width at least $k$. Let $W$ be an elementary $s$-wall so that $G$ contains some subdivision $W^{\prime}$ of $W$, where each $e \in E(W)$ has been replaced by some path $P^{e} \subseteq G$ of length $m(e)$. The outer cycle $C$ of $W$ satisfies $d_{C}(u, v) \leqslant 3 d_{W}(u, v)$ for all $u, v \in V(C)$. Moreover, $C$ is the $\mathbb{F}_{2}$-sum of cycles of length at most six.

Define a length-function $\ell$ on $G$ as follows. Let $e \in E(G)$. If $e \in P^{f}$ for $f \in E(C)$, let $\ell(e):=1 / m(f)$. Then $\ell\left(P^{f}\right)=1$ for every $f \in E(C)$. If $e \in P^{f}$ for $f \in E(W) \backslash E(C)$, let $\ell(e):=3 / m(f)$. Then $\ell\left(P^{f}\right)=3$ for every $f \in E(W) \backslash E(C)$. If $e \notin E\left(W^{\prime}\right)$, choose $\ell(e)$ large enough so that $\ell(e)>\ell\left(W^{\prime}\right)$, for example take $\ell(e):=10 s^{3}$.

It is easy to see that the subdivision $C^{\prime} \subseteq G$ of $C$ is $\ell$-geodesic in $G$. It has length $\ell\left(C^{\prime}\right)=|C| \geqslant 3 s$ and is the $\mathbb{F}_{2}$-sum of the subdivisions of 6 -cycles of $W$. Each of these has $\ell$-length 18 . Rescaling all lengths by a factor of $1 / 18$ yields the desired result.

\section{References}

[1] P. Gollin and K. Heuer. Geodesic cuts. In preparation. 
[2] Matthias Hamann and Daniel Weißauer. Bounding connected tree-width. SIAM J. Discrete Math., 30(3):1391-1400, 2016.

[3] N. Robertson and P.D. Seymour. Graph minors. II. Algorithmic aspects of tree-width. J. Algorithms, 7:309-322, 1986.

[4] N. Robertson and P.D. Seymour. Graph minors. V. Excluding a planar graph. J. Combin. Theory (Series B), 41:92-114, 1986.

[5] R. Diestel. Graph Theory. Springer, 5th edition, 2017. 